\title{
La dimensión ideológica de la enseñanza del Diseño: Riquezas y complejidades pedagógicas Proyecto de Investigación $\mathrm{N}^{\circ} 9.1$ \\ Equipo de Investigación ${ }^{(1)}$ \\ Cecilia Mazzeo
}

por Facultad de Diseño y Comunicación, Universidad de Palermo (ARG)

Resumen: El Proyecto de Investigación 9.1 presenta la problemática de la enseñanza, en todos sus niveles y contextos como una práctica compleja que involucra una multiplicidad de componentes y acciones que tienen a la Didáctica General como marco teórico de referencia. Específicamente la Didáctica Proyectual por su parte, complejiza la mirada centrándose en lo disciplinar. Dando cuenta de un nivel de complejidad diferente, la enseñanza en tanto práctica social, involucra una dimensión de naturaleza diferente, y que no suele ser considerada con la profundidad que amerita en función de su rol determinante de objetivos, contenidos y estrategias: la dimensión ideológica. Esta subyace en cada instancia del proceso e impacta tanto sobre la decisión de incluir un campo de conocimiento en el contexto académico, como sobre la construcción de planes de estudio, el diseño de programas de asignaturas y la planificación e implementación de toda propuesta de enseñanza. Reflexionar conjuntamente sobre dicha dimensión es el eje propuesto en este proyecto, en el que la mirada desde diferentes disciplinas y contextos académicos posibilita ahondar en la riqueza y complejidad de esta perspectiva de la enseñanza del diseño.

Palabras clave: Diseño - Proyecto - Enseñanza - Paradigmas - Metodología

[Resúmenes en inglés y portugués en la página 221]

${ }^{(1)}$ Los CVs del Equipo de Investigación pueden consultarse en el Capítulo Directores de Líneas y Coordinadores de Proyectos de esta misma Edición.

\section{Acerca del Proyecto 9.1}

La dimensión ideológica de la enseñanza del Diseño: Riquezas y complejidades pedagógicas

El Proyecto 9.1 La dimensión ideológica de la enseñanza del Diseño: Riquezas y complejidades pedagógicas, persigue reflexionar de manera conjunta sobre la dimensión ideoló- 
gica de la enseñanza de las disciplinas proyectuales con el objetivo de articular la mirada desde diferentes disciplinas y contextos académicos.

Avanzar en una investigación en la que participan académicos de distintas Instituciones, con la coordinación de Cecilia Mazzeo (UP), con el fin de generar un corpus de estudio sobre La dimensión ideológica de la enseñanza del Diseño que subyace en cada instancia del proceso e impacta tanto sobre la decisión de incluir un campo de conocimiento en el contexto académico, como sobre la construcción de planes de estudio, el diseño de programas de asignaturas y la planificación e implementación de toda propuesta de enseñanza. Las reflexiones y los resultados obtenidos en el Proyecto 9.1 La dimensión ideológica de la enseñanza del Diseño: Riquezas y complejidades pedagógicas se continúan desarrollando en el Proyecto 9.2 Estrategias didácticas en escenarios de innovación tecnológica: Riquezas y complejidades pedagógicas en el marco de la misma Línea de Investigación y bajo la misma Directora Cecilia Mazzeo.

Sus principales objetivos son:

- Aportar a la construcción de un corpus teórico específico.

- Instalar en el campo disciplinar un nuevo eje de producción teórica.

- Propiciar el intercambio de producciones teóricas interdisciplinares.

- Consolidar la producción teórica referida a la Didáctica Proyectual como campo específico de producción teórica.

\section{Acerca de la Línea 9}

Enseñar Disciplinas Proyectuales dirigida por Cecilia Mazzeo, se desarrolla de manera ininterrumpida desde 2015 en la Facultad de Diseño y Comunicación (UP, Argentina), e incluye hasta el momento tres proyectos finalizados el 9.1 La dimensión ideológica de la enseñanza del Diseño y 9.2 Estrategias didácticas en escenarios de innovación tecnológica, coordinados por Cecilia Mazzeo (UP), y el 9.3 Tradición e Innovación, Desafíos del Cambio Curricular: Debates, actores, contenidos y estrategias, coordinado por Cecilia Mazzeo (UP) y Guillermo Sánchez Borrero (PUCE).

\section{Mapa de Áreas y Proyectos}

El Proyecto 9.1 se vincula con todas las carreras de grado y posgrado correspondientes a la Facultad de Diseño y Comunicación. 


\section{Productos y Resultados}

\section{a)- Publicaciones}

Cuaderno del Centro de Estudios de Diseño y Comunicación N67. (2018) La dimensión ideológica de la enseñanza del Diseño. Coordinación Cecilia Mazzeo. Año XVIII, Mayo 2018, Buenos Aires, Argentina. ISSN: 1668-0227. Esta publicación documenta y comunica los resultados alcanzados en el proyecto de investigación 9.1 La dimensión ideológica de la enseñanza del Diseño, y a continuación se detallan los autores y artículos contenidos en ella: Mazzeo, Cecilia (2018) Prólogo. La dimensión ideológica de la enseñanza del Diseño (Pp. 11-17)

Necuzzi, Constanza (2018) Educación, enseñanza y didáctica en la contemporaneidad (Pp. 19-37)

Olmedo, Inés (2018) La Dirección de Arte en el cine, desafíos disciplinares y pedagógicos (Pp. 39-61)

Galán, Beatriz (2018) Reconstruyendo el entramado de una sociedad creativa. Estrategias para la formación de diseñadores en contextos de complejidad (Pp. 63-100)

Ben Altabef, Clara (2018) Intenciones para una didáctica proyectual. Caso: asignatura Proyecto y Forma en la FAU-UNT (Pp. 101-133)

Bermúdez Aguirre, Diego Giovanni (2018) El estado de posibilidad de la Historia del Diseño (Pp. 135-146)

Ledesma, María (2018) Luces y sombras en la enseñanza del Diseño. Una reflexión sobre su transformación en saber universitario (Pp. 147-162)

Cravino, Ana (2018) Enseñar Diseño: La emergencia de la teoría (Pp. 163-185)

López, Mabel Amanda (2018) Modos de decir y modos de ser: palabra e ideología en el taller de diseño (Pp. 187-213)

Romano, Ana María (2018) La construcción de la cosmovisión durante la enseñanza (Pp. 215 a 234)

Actas de Diseño No24. (2018). XII Encuentro Latinoamericano de Diseño "Diseño en Palermo". Foro de Escuelas de Diseño - XI Plenario 2017, VIII Congreso Latinoamericano de Enseñanza del Diseño. II Coloquio de Investigación y Desarrollo en Diseño Latino. Coloquio de Investigación. Julio 2017, Buenos Aires, Argentina. En esta publicación se documentan las ponencias presentadas en la Comisión de la Línea de Investigación No9: Enseñar disciplinas proyectuales en el II Coloquio de Investigación y Desarrollo en Diseño (Pp. 12-13).

Escritos en la Facultad No122. (2016) Articulación entre Investigación y Posgrados, Edición 1. Doctorado en Diseño. Maestría en Gestión del Diseño. Programa de Investigación y Desarrollo en Diseño Latino. Línea de Investigación No9: Enseñar disciplinas proyectuales. ISSN: 1669-2306. ISSN (En línea): 2591-3743. En esta publicación la Directora Cecilia Mazzeo presenta la línea de investigación $N^{\circ} 1$ Enseñar disciplinas proyectuales y anuncia el comienzo del Proyecto 9.1. La dimensión ideológica de la enseñanza del Diseño. 


\section{b)- Congresos / Coloquios / Plenarios}

$1^{\circ}$ Plenario de Directores de Investigación DC, 13 de octubre - 2017. Es este primer plenario los Directores de Líneas y Proyectos de Investigación presentaron a sus pares y al conjunto del Programa de Investigación de la Facultad de Diseño y Comunicación, los resultados obtenidos y/o en proceso (publicaciones y acuerdos), junto con los Proyectos. La Directora de la Línea de Investigación No9 presentó a sus pares, los principales avances del Proyecto 9.1 La dimensión ideológica de la enseñanza del Diseño.

II Coloquio de Investigación y Desarrollo en Diseño Latino. Universidad de Palermo, 31 de julio de 2017. VIII Congreso Latinoamericano de Enseñanza del Diseño. Semana Internacional del Diseño en Palermo.

En la comisión Enseñar disciplinas proyectuales se presentaron las reflexiones y avances del proyecto actualmente en desarrollo 9.1. La dimensión ideológica de la enseñanza del Diseño, expusieron: Cecilia Mazzeo, María Ledesma, Mabel Amanda López, Constanza Necuzzi y Ana María Romano.

A continuación se detallan las ponencias presentadas en esta Comisión coordinada por la Directora del proyecto Cecilia Mazzeo:

Mazzeo Cecilia

La dimensión ideológica de la enseñanza del Diseño

Ledesma Maria

Luces y sombras en la enseñanza del diseño. Una reflexión sobre su transformación en saber universitario

Lopez Mabel Amanda

Modos de decir y modos de ser: palabra e ideología en el taller de diseño

Necuzzi Constanza

Educación, enseñanza y didáctica en la contemporaneidad

Romano Ana María

La construcción de la cosmovisión durante la enseñanza

I Coloquio de Investigación y Desarrollo en Diseño Latino. Universidad de Palermo, 26 de Octubre de 2016.

Se presentó la Línea de Investigación No9: Enseñar disciplinas proyectuales, entramados conceptuales determinantes en la enseñanza del diseño, como una de las líneas que integran el Programa de Investigación en Diseño de la Facultad de Diseño y Comunicación.

Mazzeo Cecilia. (Argentina) Presentación de la Línea de Investigación No9: Enseñar disciplinas proyectuales. Facultad de Diseño y Comunicación, Universidad de Palermo. 


\title{
c)- Formación de Posgrado e Impacto curricular
}

La Directora Cecilia Mazzeo es Docente de Posgrado en Diseño de la Facultad de Diseño y Comunicación en Maestría en Gestión del Diseño y Doctorado en Diseño, e incorpora los contenidos de su investigación a sus asignaturas en posgrado.

\section{Evaluación Externa}

El Proyecto 9.1 La dimensión ideológica de la enseñanza del Diseño: Riquezas y complejidades pedagógicas cuyos resultados fueron publicados en el Cuaderno $\mathrm{N}^{\circ} 67$, fue evaluado exitosamente por Evaluadora Rita Aparecida da Conceição Ribeiro en 2019.

\begin{abstract}
Research Project 9.1 presents the problem of teaching, at all levels and contexts, as a complex practice that involves a multiplicity of components and actions that have General Didactics as a theoretical framework of reference. Specifically, Project Didactics, for its part, complicates the look by focusing on the disciplinary. Taking into account a different level of complexity, teaching as a social practice involves a dimension of a different nature, and which is not usually considered with the depth that it deserves based on its determining role of objectives, content and strategies: the ideological dimension. This underlies each instance of the process and impacts both on the decision to include a field of knowledge in the academic context, as on the construction of study plans, the design of subject programs and the planning and implementation of any teaching proposal. Reflecting jointly on this dimension is the axis proposed in this project, in which the look from different disciplines and academic contexts makes it possible to delve into the richness and complexity of this perspective of the teaching of design.
\end{abstract}

Keywords: Design - Project - Teaching - Paradigms - Methodology

Resumo: O Projeto de Pesquisa 9.1 apresenta o problema do ensino, em todos os níveis e contextos, como uma prática complexa que envolve uma multiplicidade de componentes e ações que têm a Didática Geral como quadro teórico de referência. Especificamente, o Project Didactics, por sua vez, complica o olhar ao focar no disciplinar. Tendo em conta um nível de complexidade diferente, o ensino como prática social envolve uma dimensão de natureza diferente, e que normalmente não é considerada com a profundidade que merece com base no seu papel determinante de objetivos, conteúdos e estratégias: a dimensão ideológica. Esta é a base de cada instância do processo e impacta tanto na decisão de inserção de uma área do conhecimento no contexto acadêmico, quanto na construção de planos de estudos, na concepção de programas de disciplinas e no planejamento e implementação de qualquer proposta de ensino. Refletir conjuntamente sobre esta 
dimensão é o eixo proposto neste projeto, em que o olhar a partir de diferentes disciplinas e contextos acadêmicos permite mergulhar na riqueza e complexidade desta perspectiva do ensino do design.

Palavras chave: Design - Projeto - Ensino - Paradigmas - Metodologia

[Las traducciones de los resúmenes fueron realizadas a través de traductor automático] 